\title{
NOISE TRANSFORMATION IN NONLINEAR SYSTEM WITH INTENSITY DEPENDENT PHASE ROTATION
}

\author{
V. V. ZVEREV* \\ Ural State Technical University, Mira 19, 620002, Ekaterinburg K-2, Russia
}

\begin{abstract}
The statistical behavior of a nonlinear system described by a mapping with phase rotation is studied. We use the Kolmogorov-Chapman equations for the multi-time probability distribution functions for investigation of dynamics under the external noise perturbations. We find a stationary solution in the long-time limit as a power series around a state with complete phase randomization ("phase mixing"). The Ornstein-Uhlenbeck and Kubo-Andersen models of noise statistics are considered; the conditions of convergence of the power expansions are established.
\end{abstract}

2000 Mathematics Subject Classification: Primary: 70K55; Secondary: 34F05, 34K50, 37H10,37L55

Keywords: Dynamical systems; Nonlinear mappings; Chaos; Markovian noise

\section{Introduction}

Noise is a key governing factor in behavior of dissipative nonlinear systems. Strictly speaking, any purely deterministic description appears to be inconsistent, because dissipative processes are connected to random kinetic motion (thermal processes, spontaneous emission, radioactive decay, etc.), so that they are stochastic in nature. Nevertheless, deterministic models provide an fair description of averaged motion for systems with stable trajectories. In the opposite case of unstable motion, random fluctuations may be amplified, so that validity of non-stochastic descriptions becomes questionable. The latter case is of particular importance, because many effects, like noise-induced transitions [1] and deterministic diffusion [2, 3], are inherent in stochastic nonlinear systems. It is also worth to mention current attempts to reconsider the background of statistical mechanics and to demonstrate that deterministic chaos underlies the origin of macroscopic irreversibility [3]-[6].

In this work we consider an evolution of a nonlinear noisy discrete dynamical system. We concentrate on evolutionary mappings with a controllable mixing, exemplified by the Ikeda mapping [7]. By this is meant that the maximal Lyapunov exponent $L$ is an increasing function of a control parameter $\lambda$ and the case $L, \lambda \gg 1$ corresponds to chaotic motion with intense phase mixing [8]. The main idea of our work is to use $\lambda^{-1}$ as a small parameter and to analyze power series around a "basic" solution corresponding to the limiting case $\lambda \rightarrow \infty$. It is important that in the limit $\lambda \rightarrow \infty$ the original nonlinear problem reduces to a linear one [8]. Analytical description of such limit transition is important by itself and it may be useful for validity check of computer simulations. As it was shown in [9, 10] for the case of intense phase mixing, the stationary solutions are associated with certain multifractal measures.

*E-mail: zverev@dpt.ustu.ru 
We present some rigorous results for a nonlinear circuit with delayed feedback, associated with the Ikeda mapping, which served as a model for radiation field dynamics in an optical ring cavity with a nonlinear medium [7, 11]. It can be viewed as a particular case of a wide class of mappings with controllable mixing. For instance, reduction of ordinary differential equations to mappings (for systems under the action of piecewise constant or delta-shaped forces) often leads to the mappings with "nonlinear phase rotations". In this situation the intense mixing occurs if a small displacement of a phase point leads to a non small "rotation" represented via an element of the orthogonal or unitary group. The 3D mapping for a nuclear magnetization system [12, 13], the 2D mapping for parametrically driven spin waves in ferromagnetic [14], the 2D mapping for a periodically kicked dissipative oscillator [15] belong to this class of mappings.

The random dynamics of the system under consideration is determined by external noise statistics. Due to the delayed feedback, the noise contributions from the different times form a superposition. For this reason it is important to preassign time correlation properties of the external noise unambiguously. Here we consider two widely used models of external noise: the Ornstein-Uhlenbeck [1] and KuboAndersen [16] random processes. Both of them may be thought of as an outcome of the Brownian motion in the thermostat with continuous or discrete assembly of states.

In the Section 2 we introduce a nonlinear model, write the generalized Kolmogorov-Chapman [17] equations for the multi-time distribution functions and derive the corresponding equations for their Fourier transforms. In the next Section, we examine the case of intense phase mixing $\lambda \rightarrow \infty$ leading to reduced equations; the stationary solution is found by applying the iterative method. In the Section 4 , we find stationary solutions for the original equations in the form of power series. In the Section 5, we prove a convergence of series solution and obtain the convergence conditions for this series. In the Section 6, we discuss a decisive role of the "rapid" Gaussian noise.

\section{A basic model}

Consider a circuit with a nonlinear element (NE) and a delayed feedback. We assume that the transformation of a slowly varying complex valued amplitude $X$ consists of the phase shift $\phi \rightarrow \phi+\lambda|X|^{2}+\Theta_{0}$, and the dissipative contraction $|X| \rightarrow \kappa|X|, \kappa<1$. The signal travels from the NE through the feedback loop (with a round-trip time $T_{d}$ ) and reaches the mixing device, where it adds to the external signal $1+\xi(t)$. Assuming $\xi(t)$ being a random process with zero mean value $\langle\xi(t)\rangle=0$, we can write the stochastic difference equation of motion

$$
\begin{aligned}
X(t) & =\xi(t)+F\left(X\left(t-T_{d}\right)\right) \\
& \equiv \xi(t)+1+\kappa X\left(t-T_{d}\right) \exp \left\{i \lambda\left|X\left(t-T_{d}\right)\right|^{2}+i \Theta_{0}\right\} .
\end{aligned}
$$

Equation (2.1) may be rewritten in the form of two-dimensional (complex) mappings family

$$
X_{N+1}=\xi_{N}+F\left(X_{N}\right),
$$

where $X_{N}=X\left(t_{0}+N T_{d}\right)$ and $\xi_{N}=\xi\left(t_{0}+(N+1) T_{d}\right), 0 \leq t_{0}<T_{d}$. We suppose that the external noise consist of two components - one is the Ornstein-Uhlenbeck random process with small correlation

time: $\tau_{c o r} \ll T_{d}$ (hereafter referred to as the "rapid" Gaussian noise); the Fourier transform of its distribution function $P_{G}(\eta)$ is given by $\Lambda(U)=\exp \left(-Q|U|^{2} / 4\right)$. A second noise component is assumed 
to be the Ornstein-Uhlenbeck or Kubo-Andersen Markovian random process with arbitrary $\tau_{c o r}$ and the transition density functions:

(i) for the Ornstein-Uhlenbeck process

$$
W(\xi, \eta,[\tau])=\left\{\pi R\left(1-\zeta_{\tau}^{2}\right)\right\}^{-1} \exp \left(-\left|\xi-\eta \zeta_{\tau}\right|^{2} / R\left(1-\zeta_{\tau}^{2}\right)\right)
$$

(ii) for the Kubo-Andersen process

$$
W(\xi, \eta,[\tau])=\zeta_{\tau} \delta^{(2)}(\xi-\eta)+q(\xi)\left(1-\zeta_{\tau}\right),
$$

where $\zeta_{\tau}=\exp \left(-\tau / \tau_{c o r}\right), q(\xi)=\sum_{k=1}^{K_{0}} p_{k} \delta^{(2)}\left(\xi-\xi_{k}\right)$ and $\delta^{(2)}(x)=\delta(\operatorname{Re} x) \delta(\operatorname{Im} x)$ is the $2 D$ (complex) delta-function. The presence of the separate "rapid" Gaussian noise component in the external signal is a decisive element in the convergence proof in Section 5 .

Note that the Kubo-Andersen random process (a special case of the kangaroo process [16]) is a stepwise constant process describing random jumps between complex values $\xi_{k}, k=1,2, \ldots, \bar{K}_{0}$ (with appearance probabilities $p_{k}$ ); switching times are uniformly and independently distributed along the time axis.

Under the above-mentioned assumptions we can write the generalized Kolmogorov-Chapman equation [8, 17] for the multi-time distribution functions in the form

$$
P_{N+1}\left(\left(X_{s}\right), \zeta\right)=\int d \bar{Y} d \xi Q\left(\left(X_{s}\right),\left(Y_{s}\right), \zeta, \xi\right) P_{N}\left(\left(Y_{s}\right), \xi\right)
$$

where

$$
\begin{aligned}
& Q\left(\left(X_{s}\right),\left(Y_{s}\right), \xi_{n+1}, \xi_{0}\right)=\int d \bar{Y} d \bar{\eta} d \xi_{1} \cdots d \xi_{n} \prod_{k=0}^{n} P_{G}\left(\eta_{k}\right) \\
& \times \delta^{(2)}\left(X_{k}-\xi_{k}-\eta_{k}-F\left(Y_{k}\right)\right) \quad W\left(\xi_{k+1}, \xi_{k},\left[\tau_{k+1}-\tau_{k}\right]\right) .
\end{aligned}
$$

In Eq. 2.5) we used a short-hand notation

$$
P_{N}\left(\left(X_{s}\right), \xi\right) \equiv P\left(X_{0}\left[N T_{d}+\tau_{0}\right], X_{1}\left[N T_{d}+\tau_{1}\right], \ldots, X_{n}\left[N T_{d}+\tau_{n}\right], \xi\left[N T_{d}\right]\right),
$$

where $X_{i}, i=0,1, \ldots, n$, are the signal amplitudes at $N T_{d}+\tau_{i}$, respectively, and $\xi$ is the amplitude of the second noise component at $N T_{d}$. We also require $0 \equiv \tau_{0}<\tau_{1}<\ldots<\tau_{n+1} \equiv T_{d}$ and use a notation $d \bar{A}=d A_{0} d A_{1} \ldots d A_{n}$.

The corresponding equation for the distribution function Fourier transform

$$
\Psi_{N}\left(\left(U_{s}\right), \Omega\right)=\int d \bar{X} d \xi P_{N}\left(\left(X_{s}\right), \xi\right) \exp \left\{-i \operatorname{Re}\left(\sum_{k=0}^{n} X_{k} U_{k}^{*}+\xi \Omega^{*}\right)\right\}
$$

is given by

$$
\Psi_{N+1}\left(\left(U_{s}\right), \Omega\right)=\int d \bar{V} d \theta \Upsilon\left(\left(U_{s}\right),\left(V_{s}\right), \Omega, \theta\right) \Psi_{N}\left(\left(V_{s}\right), \theta\right)
$$

where 


$$
\begin{gathered}
\Upsilon\left(\left(U_{s}\right),\left(V_{s}\right), \Omega, \theta\right)=Z\left(\left(U_{s}\right), \Omega, \theta\right) \prod_{k=0}^{n} \Lambda\left(U_{k}\right) \sigma\left(U_{k}, V_{k}\right), \\
\sigma(U, V)=(2 \pi)^{-2} \int d Y \exp \left\{i \operatorname{Re}\left(Y V^{*}-F(Y) U^{*}\right)\right\}, \\
Z\left(\left(U_{s}\right), \Omega_{n+1}, \Omega_{0}\right)=\int d \Omega_{1} \ldots d \Omega_{n} \prod_{k=0}^{n} H\left(\Omega_{k+1}, \Omega_{k}-U_{k},\left[\tau_{k+1}-\tau_{k}\right]\right), \\
H(\Omega, \Gamma,[\tau])=(2 \pi)^{-2} \int d \xi d \eta W(\xi, \eta,[\tau]) \exp \left\{i \operatorname{Re}\left(\eta \Gamma^{*}-\xi \Omega^{*}\right)\right\} .
\end{gathered}
$$

Subscripts replacement $N, N+1 \rightarrow$ st reduces Eqs.2.5) and (2.9) to the equations for the stationary distributions:

$$
\begin{aligned}
& P_{s t}\left(\left(X_{s}\right), \zeta\right)=\int d \bar{Y} d \xi Q\left(\left(X_{s}\right),\left(Y_{s}\right), \zeta, \xi\right) P_{s t}\left(\left(Y_{s}\right), \xi\right), \\
& \Psi_{s t}\left(\left(U_{s}\right), \Omega\right)=\int d \bar{V} d \theta \Upsilon\left(\left(U_{s}\right),\left(V_{s}\right), \Omega, \theta\right) \Psi_{s t}\left(\left(V_{s}\right), \theta\right) .
\end{aligned}
$$

\section{Intense phase mixing case}

The expression 2.11) may be presented in the form 8

$$
\sigma=\tilde{\sigma}+\Delta \sigma
$$

where

$$
\begin{aligned}
\sigma(U, V)=(2 \pi|V|)^{-1} & e^{-i \operatorname{Re} U} \delta^{(2)}(|V|-\kappa|U|), \Delta \sigma(U, V)=e^{-i \operatorname{Re} U} \beta(U, V), \\
\beta(U, V)= & \frac{1}{2 \pi \lambda} \sum_{\nu=1}^{\infty} \frac{1}{\nu} J_{\nu}\left(\frac{\kappa|U V|}{2 \lambda \nu}\right) \\
& \times \cos \left\{\nu\left(\Theta_{0}+\arg \left(\frac{V}{U}\right)\right)-\frac{|V|^{2}+\kappa^{2}|U|^{2}}{4 \lambda \nu}+\frac{(\nu+1) \pi}{2}\right\} .
\end{aligned}
$$

In the limit $\lambda \rightarrow \infty$ one can neglect the last term in Eq.(3.16), in other words, replace $\sigma$ by $\tilde{\sigma}$ in (2.10) (corresponding stationary solutions of Eqs.(2.14), (2.15) will be labeled by superscript ${ }^{(0)}$ ). Consider the special case of the Ornstein-Uhlenbeck random process in detail; the Kubo-Andersen process is analized the same way. Using the explicit form of the function (2.13) for the Ornstein-Uhlenbeck process:

$$
H(\Omega, \Gamma,[\tau])=\exp \left\{-\frac{1}{4}|\Omega|^{2} R\left(1-\zeta_{\tau}^{2}\right)\right\} \delta^{(2)}\left(\Gamma-\Omega \zeta_{\tau}\right),
$$

one can reduce Eq.(2.15) to 


$$
\Psi_{s t}^{(0)}=\hat{\mathbf{K}} \Psi_{s t}^{(0)}
$$

where

$$
\begin{array}{r}
\hat{\mathbf{K}} f\left(\left(U_{s}\right), U_{n+1}\right)=\int d \bar{V} d \theta f\left(\left(V_{s}\right), \theta\right) Z\left(\left(U_{s}\right), U_{n+1}, \theta\right) \prod_{k=0}^{n} \Lambda\left(U_{k}\right) \tilde{\sigma}\left(U_{k}, V_{k}\right)= \\
\quad=\prod_{k=0}^{n}\left[\Lambda\left(U_{k}\right) e^{-i \operatorname{Re} U_{k}}\right] \Phi\left(\left(U_{s}\right), U_{n+1}\right)\left\langle f\left(\left(\kappa U_{s} e^{i \phi_{s}}\right), \sum_{j=0}^{n+1} \zeta_{\tau_{j}} U_{j}\right)\right\rangle_{\left(\phi_{s}\right)},
\end{array}
$$

and

$$
\begin{gathered}
\Phi\left(\left(U_{s}\right), U_{n+1}\right)=\exp \left\{-\frac{R}{4} \sum_{k=1}^{n+1}\left|\sum_{j=k}^{n+1} \frac{\zeta_{\tau_{j}}}{\zeta_{\tau_{k}}} U_{j}\right|^{2}\left(1-\frac{\zeta_{\tau_{k}}^{2}}{\zeta_{\tau_{k-1}}^{2}}\right)\right\}, \\
\zeta_{\tau_{k}}=e^{-\tau_{k} / \tau_{c o r}}, \quad\langle\ldots\rangle_{(\phi)} \equiv \frac{1}{2 \pi} \int_{0}^{2 \pi}(\ldots) d \phi .
\end{gathered}
$$

The angular brackets in Eq.(3.21) denote an averaging over the phase angles $\phi_{k}, k=0,1, \ldots, n$. Define a linear operator $\hat{\mathbf{L}}$ acting on the vector $\left[\left(U_{s}\right), \Omega\right] \equiv\left[U_{0}, \ldots, U_{n}, \Omega\right]$ as follows

$$
\hat{\mathbf{L}}\left(\phi_{0}, \ldots, \phi_{n}\right)\left[\left(U_{s}\right), \Omega\right]=\left[\left(\kappa U_{s} e^{i \phi_{s}}\right), \sum_{k=0}^{n} \zeta_{\tau_{k}} U_{k}+\zeta_{\tau_{n+1}} \Omega\right]
$$

It is easy to show that

$$
\left\{\prod_{p=1}^{m} \hat{\mathbf{L}}\left(\phi_{0 p}, \ldots, \phi_{n p}\right)\right\}\left[\left(U_{s}\right), \Omega\right]=\left[\left(\kappa^{m} U_{s} \exp \left\{i \sum_{p=1}^{m} \phi_{s p}\right\}\right), \sum_{k=0}^{n} d_{k m} U_{k}+\zeta_{\tau_{n+1}}^{m} \Omega\right]
$$

where

$$
d_{k m}=\zeta_{\tau_{k}} \sum_{q=0}^{m-1} \kappa^{q} \zeta_{\tau_{n+1}}^{m-1-q} \exp \left\{i \sum_{p=1}^{q} \phi_{k p}\right\}
$$

and $\phi_{k p}$ are arbitrary angles: $0 \leq \phi_{k p}<2 \pi, 1 \leq p \leq m, 0 \leq k \leq n$ (the operators in the product in Eq.(3.24) are enumerated from right to left). One can show that

$$
\left|d_{k m}\right| \leq \zeta_{\tau_{k}} \sum_{q=0}^{m-1} \kappa^{q} \zeta_{\tau_{n+1}}^{m-1-q} \leq \zeta_{\tau_{k}} \cdot m\left(\max \left\{\kappa, \zeta_{\tau_{n+1}}\right\}\right)^{m-1 \stackrel{m \rightarrow \infty}{\longrightarrow} 0}
$$

As consequence, we have 


$$
\lim _{m \rightarrow \infty}\left\{\prod_{p=1}^{m} \hat{\mathbf{L}}\left(\phi_{0 p}, \ldots, \phi_{n p}\right)\right\}\left[\left(U_{s}\right), \Omega\right]=[(0), 0]
$$

and

$$
\lim _{m \rightarrow \infty} \hat{\mathbf{K}}^{m} f\left(\left(U_{s}\right), \Omega\right)=f((0), 0) \cdot \lim _{m \rightarrow \infty} \hat{\mathbf{K}}^{m} \mathbf{1}
$$

for a continuous function $f$ (in the r.h.s. of Eq. 3.27 ) the operator acts to the right onto the real constant equal to unity). With help of the expression (3.27), using Eq.(3.20) in the iteration procedure

$$
\Psi_{s t}^{(0)}=\hat{\mathbf{K}} \Psi_{s t}^{(0)}=\hat{\mathbf{K}}^{2} \Psi_{s t}^{(0)}=\ldots,
$$

and taking into account the normalization condition

$$
\Psi_{s t}^{(0)}((0), 0)=\int d \bar{X} d \xi P_{s t}^{(0)}\left(\left(X_{s}\right), \xi\right)=1,
$$

we can write a solution of Eq.(3.20) in a simple form

$$
\Psi_{s t}^{(0)}\left(\left(U_{s}\right), \Omega\right)=\lim _{m \rightarrow \infty} \hat{\mathbf{K}}^{m} \mathbf{1}
$$

Note that the solution (3.30) is the asymptotically stable "fixed point" of the evolutionary mapping

$$
\Psi_{N+1}^{(0)}=\hat{\mathbf{K}} \Psi_{N}^{(0)},
$$

so that it is independent of initial conditions. This reflects decay of correlations and "memory loss" in the system. The transformation $P_{N} \rightarrow P_{N+1}$ defined by Eq.(2.5) preserves the norm for arbitrary $\lambda$. Consequently, the condition $|f| \leq 1$ implies $|\hat{\mathbf{K}} f| \leq 1$, so that absolute values of (3.27) and (3.30) are bounded from above. The operator $\hat{\mathbf{K}}$ performs transformations including dilation $\left|U_{s}\right| \rightarrow \kappa\left|U_{s}\right|$ and averaging over the $n$-dimensional torus. It is remarkable that we will have Eq. (3.31) in the place of Eq.(2.9) if we substitute the linear stochastic difference equation

$$
X(t)=\xi(t)+1+\kappa X\left(t-T_{d}\right) \exp \{i \tilde{\theta}(t)\}
$$

instead of the nonlinear Eq. 2.1); here $\tilde{\theta}(t)$ is the random process with independent and uniformly distributed values. As it was shown in our work [10], the solutions of a type (3.30) are associated with certain integrals over multifractals.

Using statistical moments approach, one may arrive at the same results in other way [8]. Define the stationary moments of distributions as follows:

$$
M_{\gamma}^{s t}=\left\langle\xi^{p} \xi^{* q} \prod_{k=0}^{n}\left(X_{k}^{r_{k}} X_{k}^{* l_{k}}\right)\right\rangle_{s t}=D_{\gamma}\left(\Psi_{s t}^{(0)}\right),
$$

where multiple derivatives

$$
\left.D_{\gamma}(f) \stackrel{\text { def }}{=} \frac{\partial^{p}}{\partial \Omega^{* p}} \frac{\partial^{q}}{\partial \Omega^{q}} \prod_{k=0}^{n}\left(\frac{\partial^{r_{k}}}{\partial U_{k}^{* r_{k}}} \frac{\partial^{l_{k}}}{\partial U_{k}^{l_{k}}}\right) f\left(\left(U_{s}\right), \Omega\right)\right|_{\left(U_{s}\right)=\mathbf{0}, \Omega=0}
$$


are computed at the origin, and $\gamma=\left(p, q,\left(r_{s}, l_{s}\right)\right)$ denotes the multi-index. It follows from Eq. (3.21) that $D_{\gamma}(\hat{\mathbf{K}} f)$ is a linear combination of $D_{\gamma^{\prime}}(f)$ for $\left|\gamma^{\prime}\right|<|\gamma|$ (here we denote $|\gamma|=p+q+\sum_{k=0}^{n}\left(r_{k}+l_{k}\right)$ ). Introduce a vector $\mathbf{m}(f)$ of values $D_{\gamma}(f),|\gamma|<\left|\gamma_{\max }\right|$, and choose special ordering of its components: provided that either $\left|\gamma_{2}\right|<\left|\gamma_{1}\right|$, or $\left|\gamma_{2}\right|=\left|\gamma_{1}\right|$ with $p_{2}+q_{2}>p_{1}+q_{1}, D_{\gamma_{1}}(f)$ precedes $D_{\gamma_{2}}(f)$ :

$$
\mathbf{m}(f)=\left(\ldots, D_{\gamma_{1}}(f), \ldots, D_{\gamma_{2}}(f), \ldots\right) .
$$

It is easy to see that

$$
\mathbf{m}(\hat{\mathbf{K}} f)=\mathbf{a m}(f)+\mathbf{b}, \quad \mathbf{b}=\mathbf{b}_{0} \cdot f((0), 0),
$$

where $\mathbf{a}$ is a triangular matrix. The diagonal elements

$$
\mathbf{a}_{\gamma \gamma}=\Xi_{p q} \prod_{k=0}^{n} \delta_{r_{k} l_{k}} \kappa^{r_{k}+l_{k}}
$$

with $\Xi_{p q}=e^{-T_{d} / \tau_{c o r}}+\left(1-e^{-T_{d} / \tau_{c o r}}\right) \delta_{p 0} \delta_{q 0}$ for the Kubo-Andersen process and $\Xi_{p q}=e^{-T_{d}(p+q) / \tau_{c o r}}$ for the Ornstein-Uhlenbeck process, are real and $0<\mathbf{a}_{\gamma \gamma}<1$, so that both $\mathbf{a}$ and $\mathbf{1}-\mathbf{a}$ are nonsingular matrices. Furthermore, $\mathbf{a}=\mathbf{T} \mathbf{J} \mathbf{T}^{-1}$, where $\operatorname{det} \mathbf{T} \neq 0$ and $\mathbf{J}$ is the Jordan form of the matrix $\mathbf{a}$ (each diagonal element of $\mathbf{J}$ also is positive and less than unity). In this case $\mathbf{a}^{m}=\mathbf{T} \mathbf{J}^{m} \mathbf{T}^{-1} \stackrel{m \rightarrow \infty}{\longrightarrow} 0$ and

$$
\begin{aligned}
& \lim _{m \rightarrow \infty}\left\{\mathbf{m}\left(\hat{\mathbf{K}}^{m} f\right)-(\mathbf{1}-\mathbf{a})^{-1} \mathbf{b}_{0} \cdot f((0), 0)\right\} \\
= & \lim _{m \rightarrow \infty} \mathbf{a}^{m}\left\{\mathbf{m}(f)-(\mathbf{1}-\mathbf{a})^{-1} \mathbf{b}_{0} \cdot f((0), 0)\right\}=0 .
\end{aligned}
$$

Matrix relations (3.36) for various $\gamma_{\max }$ present another equivalent form of the condition (3.27).

\section{Series expansion of exact solution}

In the general case of arbitrary values of $\lambda$ we can rewrite Eq.(2.15) as

$$
\Psi_{s t}=(\hat{\mathbf{K}}+\varepsilon \hat{\mathbf{S}}) \Psi_{s t},
$$

where $\varepsilon$ is an auxiliary parameter. We are seeking a solution of Eq. 4.39) in the form of formal power series in $\varepsilon$ [18]:

$$
\Psi_{s t}=\sum_{j=0}^{\infty} \varepsilon^{j} \Psi_{s t}^{(j)}
$$

with normalization conditions

$$
\begin{gathered}
\Psi_{s t}((0), 0)=\Psi_{s t}^{(0)}((0), 0)=1, \\
\Psi_{s t}^{(j)}((0), 0)=0, \quad j>0 .
\end{gathered}
$$

Substituting the expansion (4.40) into Eq.4.39) and equating coefficients of like powers of $\varepsilon$, we obtain Eq.(3.20) and 


$$
\Psi_{s t}^{(j)}=\hat{\mathbf{K}} \Psi_{s t}^{(j)}+\hat{\mathbf{S}} \Psi_{s t}^{(j-1)} .
$$

Repeated application of the last relation yields

$$
\Psi_{s t}^{(j)}=\hat{\mathbf{K}}^{m} \Psi_{s t}^{(j)}+\sum_{p=0}^{m-1} \hat{\mathbf{K}}^{p} \hat{\mathbf{S}} \Psi_{s t}^{(j-1)}
$$

where $m$ is an arbitrary positive integer. In the limit $m \rightarrow \infty$ in Eq.(4.44), using Eqs.(3.27), (4.42) and its corollary:

$$
\lim _{m \rightarrow \infty} \hat{\mathbf{K}}^{m} \Psi_{s t}^{(j)}=0, \quad j>0
$$

one can find a recursive relation

$$
\Psi_{s t}^{(j)}=\sum_{p=0}^{\infty} \hat{\mathbf{K}}^{p} \hat{\mathbf{S}} \Psi_{s t}^{(j-1)}
$$

Finally, combination of recursions 4.46) with different $j$ and substitution into Eq.(4.40) yields

$$
\Psi_{s t}=\Psi_{s t}^{(0)}+\sum_{j=1}^{\infty} \varepsilon^{j}\left\{\left(\sum_{p=0}^{\infty} \hat{\mathbf{K}}^{p}\right) \hat{\mathbf{S}}\right\}^{j} \Psi_{s t}^{(0)} .
$$

In the case $\lambda \gg 1$ the term with $\varepsilon^{j}$ in Eq.4.47) is $O\left(\lambda^{-2 j}\right)$; note that one must set $\varepsilon=1$ in the final expression.

\section{A proof of convergence}

In this section we find a condition of existence of the expansion (4.47). Consider the associated equation for the "fixed point" $\Psi_{s t}$ :

$$
\Psi_{s t}=\hat{\mathbf{A}} \Psi_{s t},
$$

for the operator:

$$
\hat{\mathbf{A}} f=\Psi_{s t}^{(0)}+\left\{\sum_{p=0}^{\infty} \hat{\mathbf{K}}^{p}\right\} \hat{\mathbf{S}} f .
$$

The operator $\hat{\mathbf{K}}$ is defined by Eq. (3.21) and

$$
\hat{\mathbf{S}} f\left(\left(U_{s}\right), \Omega\right)=\prod_{k=0}^{n} \Lambda\left(U_{k}\right) \int d \bar{V} d \theta f\left(\left(V_{s}\right), \theta\right) Z\left(\left(U_{s}\right), \Omega, \theta\right) \alpha\left(\left(U_{s}\right),\left(V_{s}\right)\right)
$$

where

$$
\alpha\left(\left(U_{s}\right),\left(V_{s}\right)\right)=\sum_{k=0}^{n} \frac{1}{(k+1) !(n-k) !} \sum_{\left(r_{s}\right)}\left[\prod_{i=0}^{k} \Delta \sigma\left(U_{r_{i}}, V_{r_{i}}\right) \prod_{j=k+1}^{n} \tilde{\sigma}\left(U_{r_{j}}, V_{r_{j}}\right)\right]
$$


The inner summation in (5.51) is taken over all the permutations

$$
\left(\begin{array}{ccccc}
0 & 1 & 2 & \ldots & n \\
r_{0} & r_{1} & r_{2} & \ldots & r_{n}
\end{array}\right)
$$

For $k=n$ the right-hand product of factors $\tilde{\sigma}$ in (5.51) is equal to unity. We are going to prove that $\hat{\mathbf{A}}$ is the contraction operator in an appropriate complete metric space. The condition of contraction is given by

$$
\left\|\hat{\mathbf{A}} f_{2}-\hat{\mathbf{A}} f_{1}\right\| \leq \alpha_{c o n}\left\|f_{2}-f_{1}\right\|, \quad \alpha_{c o n}<1 .
$$

According to the contraction mapping principle, Eq.(5.48) has the unique solution if the condition (5.52) is satisfied. From Eq.(5.49) follows

$$
\hat{\mathbf{A}} f_{2}-\hat{\mathbf{A}} f_{1}=\left\{\sum_{p=0}^{\infty} \hat{\mathbf{K}}^{p}\right\} \hat{\mathbf{S}}\left(f_{2}-f_{1}\right)
$$

and the operator in the right hand side of (5.53) is linear. In this case the condition (5.52) takes place simultaneously with the limiting condition for the operator norm

$$
\left\|\left\{\sum_{p=0}^{\infty} \hat{\mathbf{K}}^{p}\right\} \hat{\mathbf{S}}\right\| \stackrel{\text { def }}{=} \sup _{f \neq 0}\left(\frac{1}{\|f\|}\left\|\left\{\sum_{p=0}^{\infty} \hat{\mathbf{K}}^{p}\right\} \hat{\mathbf{S}} f\right\|\right)<\alpha_{\text {con }}<1 .
$$

The expansion (4.47) is convergent under the condition (5.54). On the other hand, (4.47) is the solution of Eq. (4.39) resulting from the iterative procedure.

In this work we use the norm

$$
\|f\| \stackrel{\text { def }}{=} \sup _{\left(\left(U_{s}\right), \Omega\right) \in \mathbf{C}^{n+2}}\left\{\left|f\left(\left(U_{s}\right), \Omega\right)\right| \prod_{k=0}^{n} \mu\left(U_{k}\right)\right\}
$$

with

$$
\mu(U)=\{\Lambda(U)\}^{-1 / 2}=\exp \left\{\frac{1}{8} Q|U|^{2}\right\} .
$$

(properties of this norm are discussed in the Appendix). To prove the inequalities (5.52), (5.54) and make estimate of $\alpha_{c o n}$, we have to make a number of auxiliary estimations. Using Eq.(3.19) and the similar expression for the Kubo-Andersen process:

$$
H(\Omega, \Gamma,[\tau])=\zeta_{\tau} \delta^{(2)}(\Omega-\Gamma)+\left(1-\zeta_{\tau}\right) \chi(\Omega) \delta^{(2)}(\Gamma),
$$

where

$$
\chi(\Omega)=\sum_{k=1}^{K_{0}} p_{k} \exp \left\{-i \operatorname{Re}\left(\xi_{k} \Omega^{*}\right)\right\}
$$

(see Eq.(2.4), one can find for both noise statistics

$$
\sup _{\Omega \in \mathbf{C}}\left|\int d \theta H(\Omega, \theta-U,[\tau]) f(U, \theta)\right| \leq \sup _{\Omega \in \mathbf{C}}|f(U, \Omega)|,
$$




$$
\sup _{\Omega \in \mathbf{C}}\left|\int d \theta Z\left(\left(U_{s}\right), \Omega, \theta\right) f\left(\left(U_{s}\right), \theta\right)\right| \leq \sup _{\Omega \in \mathbf{C}}\left|f\left(\left(U_{s}\right), \Omega\right)\right| ;
$$

$f(U, \Omega)$ in 5.57$)$ and $f\left(\left(U_{s}\right), \Omega\right)$ in (5.58) are arbitrary bounded complex functions. Next, using (3.21), we can obtain

$$
\sup _{\Omega \in \mathbf{C}}\left|\hat{\mathbf{K}}^{j} f\left(\left(U_{s}\right), \Omega\right)\right| \leq \prod_{k=0}^{n} \prod_{m=0}^{j-1} \Lambda\left(\kappa^{m} U_{k}\right)\left\langle\sup _{\Omega \in \mathbf{C}}\left|f\left(\left(\kappa^{j} U_{s} e^{i \phi_{s}}\right), \Omega\right)\right|\right\rangle_{\left(\phi_{s}\right)} .
$$

Besides, using (3.18) and the inequalities $\left|J_{\nu}(z)\right| \leq \frac{1}{\nu !}|z / 2|^{\nu}$ for real values of $z$ and integer positive $\nu$ [19]; $\kappa^{\nu(j+1)} \leq \kappa^{j+1}, \nu^{-\nu-1} \leq 1$ for $\nu \geq 1, j \geq 0, \kappa<1$, we can find the upper bounds

$$
\left\langle\left|\beta\left(\kappa^{j} U e^{i \phi}, V\right)\right|\right\rangle_{(\phi)} \leq \frac{\kappa^{j+1}}{2 \pi \lambda} \exp \left\{\frac{|U V|}{4 \lambda}\right\} \leq \frac{\kappa^{j+1}}{2 \pi \lambda} \exp \left\{\frac{|U|^{2}+|V|^{2}}{8 \lambda}\right\} .
$$

Finally, the inequalities (5.59), (5.60) are combined to produce the contraction condition (5.52) with

$$
\alpha_{\text {con }}=\frac{1}{1-\kappa}\left\{\left(\frac{4 \kappa}{Q \lambda-1}+1\right)^{n+1}-1\right\} .
$$

The parameter $\alpha_{\text {con }}$ is less than unity for any chosen $n$ if $Q \lambda$ is sufficiently large. Under this condition, the solution (4.47) of Eq. (2.15) exists as the unique "fixed point" of the contraction operator (5.49). It is possible to prove convergence of (4.47) in another way, using the technique of a majorizing series, but it's very cumbersome and we omit it here.

\section{Conclusions and discussion}

We have found a stationary solution to the generalized Kolmogorov-Chapman equation for the multitime distribution functions in the form of a convergent series expansion (4.47). Our approach is based on successive application of two iterative procedures. The final expressions were obtained for the case of the nonlinear Ikeda mapping and certain fluctuation statistics. This result may be used for calculation of the approximate expressions for probability distributions, statistical moments and correlation functions.

As simple example consider a case with the "rapid" Gaussian noise component only. In this case Eq.(2.15) for the Fourier transform of the one-time distribution function takes the form:

$$
\Psi_{s t}(U)=\Lambda(U) \int d V \Psi_{s t}(V) \sigma(U, V)
$$

Using the general expression (4.47), we can write approximate first-order solution as follows:

$$
\begin{gathered}
\Psi_{s t}(U) \cong \Psi_{s t}^{(0)}(U)+\left\{\sum_{p=0}^{\infty} \hat{\mathbf{K}}^{p}\right\} \hat{\mathbf{S}} \Psi_{s t}^{(0)}(U)=e^{-i \operatorname{Re} U} \Lambda(U)\left\{\eta_{\infty}(\kappa|U|)\right. \\
\left.+\int_{0}^{\infty} v d v \Lambda(v) \eta_{\infty}(\kappa v)\left(\beta_{1}(U, v)+\sum_{p=1}^{\infty} \Lambda\left(\kappa^{p} U\right) \eta_{p-1}(\kappa|U|) \beta_{2}\left(\kappa^{p}|U|, v\right)\right)\right\},
\end{gathered}
$$


where

$$
\begin{gathered}
\beta_{1}(U, v)=2 \pi\left\langle\beta\left(U, v e^{i \phi}\right) \exp \{-i v \cos \phi\}\right\rangle_{(\phi)}, \\
\beta_{2}(u, v)=2 \pi\left\langle\beta\left(u e^{i \phi_{1}}, v e^{i \phi_{2}}\right) \exp \left\{-i\left(u \cos \phi_{1}+v \cos \phi_{2}\right)\right\}\right\rangle_{\left(\phi_{1}, \phi_{2}\right)}, \\
\eta_{k}(u)=\prod_{j=0}^{k-1} \Lambda\left(\kappa^{j} u\right) J_{0}\left(\kappa^{j} u\right), \quad \eta_{0}=1, \quad \eta_{\infty}=\lim _{k \rightarrow \infty} \eta_{k} .
\end{gathered}
$$

In general case the resulting formulae are cumbersome. Nevertheless, they provide a basis for development of computational procedures (in particular, using symbolic processors). The detailed study of this applications is beyond the scope of this article.

Consider a specific role of the "rapid" Gaussian noise in our method. In order to simplify consideration, we suppose that this is the only noise component present in the system. To analize influence of noise, we replace $\sigma(U, V)$ by $\Lambda(U) \sigma(U, V)$ in Eq. 6.62). Since $\Lambda(U)=\exp \left(-\frac{1}{4} Q|U|^{4}\right)$, the Fourier spectrum becomes truncated in the region $|U| \gg 1$. It ensures the convergence of the expansion (4.47). On the other hand, as it was shown in [8], this replacement leads to a "coarse-grained" dynamics, because fluctuations coarsen the fine-scale structure of a chaotic attractor. If $Q \lambda$ is sufficiently large, the condition of contraction (5.52) may be satisfied for any $n$, so that corresponding expansions (4.40), (4.47) converge. However, we can consider the limits $\lambda \rightarrow \infty$ and $Q \rightarrow 0$ separately. The limits do not commute, so that we should set $Q \rightarrow 0$ after using $\lambda \rightarrow \infty$. It seems plausible that the correct description of the random behavior in the limit $\lambda \rightarrow \infty$ may be reached only under the action of an infinitesimal noise.

It is instructive to draw a parallel between the above manipulations and the well-known formalism of quantum mechanics. In order to obtain the retarded (causal) solutions of the Schrodinger equation, one have to write the equation containing the additional source term:

$$
\ddot{\psi}_{\epsilon}+i\left(\hat{\mathbf{H}}_{0}+\hat{\mathbf{V}}\right) \psi_{\epsilon}=-\epsilon\left(\psi_{\epsilon}-\Phi\right),
$$

where $\Phi$ is a solution for the "nonperturbed" system $(\hat{\mathbf{V}}=0)$, and further to set $\epsilon \rightarrow 0$ after the volume grows infinitely (this is the boundary condition of Gell-Mann and Goldberger [20] in the form proposed in 21]; here $\hbar=0$ ). The same approach appears to be natural to formulate one well-known version of the so-called "master equation" formalism in statistical physics. Following the technique of [21], the final equation of motion for the quasi-equilibrium statistical operator may be derived from the Liouwille equation (completed with the source term) by substituting $\epsilon \rightarrow 0$ after the thermodynamic limit is reached. Thus, in order to obtain a physically significant irreversible solution, one must add the infinitesimal dissipative term (in other words, to break the symmetry with respect to $t \rightarrow-t$ ).

Both above-mentioned procedures lead to a "coarsened" description, presented by "coarsened" equations. It is worth to note that dissipative processes accompanied by fluctuations bring into existence a strange attractor the same time destroying a fine structure of the attractor at $\lambda \rightarrow \infty$ (for an example of the attractor in our model, see [22]) .

\section{Appendix}

In this work we use a nonstandard metrics, so it is necessary to check the completeness of the respective metric space. Define a norm and a distance function on a set of functions of $n$ complex arguments 
$\mathbf{x}=\left(x_{1}, x_{2}, \ldots, x_{n}\right) \in \mathbf{C}^{n}$ as follows:

$$
\|f\|_{\infty}=\sup _{\mathbf{x} \in \mathbf{C}^{n}}|f(\mathbf{x})|, \quad \rho_{\infty}\left(f_{1}, f_{2}\right)=\left\|f_{1}-f_{2}\right\|_{\infty} .
$$

Using the standard approach (see, for example, 23], Theorem I.23), we can prove

(i) if $f_{k}(\mathbf{x}), k \in \mathbf{N}$, is a Cauchy sequence, the pointwise and uniform convergence takes place:

$$
\left(\forall \mathbf{x} \in \mathbf{C}^{n}\right)(\forall \varepsilon>0)\left(\exists N_{\varepsilon}\right)\left(\forall k>N_{\varepsilon}\right):\left|f_{k}(\mathbf{x})-f(\mathbf{x})\right|<\varepsilon
$$

(ii) if $f_{k}(\mathbf{x})$ is continuous for each $k \in \mathbf{N}, f(\mathbf{x})$ is continuous as well.

In addition, we can prove:

(iii) if all $f_{k}(\mathbf{x})$ are uniformly bounded

$$
(\exists M)(\forall k \in \mathbf{N})\left(\forall \mathbf{x} \in \mathbf{C}^{n}\right):\left|f_{k}(\mathbf{x})\right| \leq M,
$$

then $|f(\mathbf{x})| \leq M$.

It is convenient to prove (iii) by reduction to contradiction. Assume that

$$
\left(\exists \mathbf{x}_{0} \in \mathbf{C}^{n}\right):\left|f\left(\mathbf{x}_{0}\right)\right|=M+\bar{\varepsilon}, \quad \bar{\varepsilon}>0 .
$$

Choosing $\varepsilon<\bar{\varepsilon}$ and $\bar{k}$ sufficiently large to have $\left|f_{\bar{k}}\left(\mathbf{x}_{0}\right)-f\left(x_{0}\right)\right|<\varepsilon$, we arrive at a contradiction to the original assumption (A.71):

$$
\left|f_{\bar{k}}\left(\mathbf{x}_{0}\right)\right| \geq|| f\left(\mathbf{x}_{0}\right)|-| f_{\bar{k}}\left(\mathbf{x}_{0}\right)-f\left(\mathbf{x}_{0}\right)|| \geq M+\bar{\varepsilon}-\varepsilon>M .
$$

It means that the continuous uniformly bounded functions, equipped with the norm and metric A.68), form the complete metric space.

It follows that the space $C^{\star} \subset C\left(\mathbf{C}^{n+2}\right)$ of continuous functions, satisfying

$$
\left|f\left(\left(U_{s}\right), \Omega\right)\right| \leq M \prod_{k=0}^{n} \mu^{-1}\left(U_{k}\right)
$$

with the norm (5.55) and the distance $\rho_{C \star}\left(f_{1}, f_{2}\right)=\left\|f_{1}-f_{2}\right\|$, is the complete metric space (the condition (A.73) is rewritten as $\|f\| \leq M$ ).

Finally, note that $f \in C^{\star}$ implies $A f \in C^{\star}$. Choosing a class of functions with $\|f\| \leq M$, where $M$ is sufficiently large: $M>\left(1-\alpha_{c o n}\right)^{-1}$ (the functional space $C^{\star}$ may be expanded by replacement $M \rightarrow M^{\prime}, M^{\prime}>M$, if required), we may find that

$$
\|A f\| \leq\left\|\Psi_{s t}^{(0)}\right\|+\left\|\left(\sum_{p=0}^{\infty} \hat{\mathbf{K}}^{p}\right) \hat{\mathbf{S}} f\right\| \leq 1+\alpha_{\text {con }}\|f\| \leq 1+\alpha_{\text {con }} M<M .
$$

\section{Acknowledgments}

The author wants to thank B.Y. Rubinstein for help and discussions. The work was supported in part by grant of RF State Committee on High Education 95-0-8.3-14. 


\section{References}

[1] W.Horsthemke, R. Lefever, Noise-Induced Transitions. Theory and Applications in Physics, Chemistry and Biology, in Springer Series in Synergetics, 15, ed. H. Haken (Springer, 1984).

[2] P. Gaspard, R. Klages, Chaotic and Fractal Properties of Deterministic Diffusion-Reaction Processes, Chaos 8 (1998) 409-423.

[3] T. Gilbert, J.R. Dorfman, P. Gaspard, Entropy Production, Fractals, and Relaxation to Equilibrium, Physical Review Letters 85 (2000) 1606-1609.

[4] J.L. Lebowitz, Microscopic Origins of Irreversible Macroscopic Behavior, Physica A263 (1999) 516-527.

[5] I. Prigogine, Laws of Nature, Probability and Time Symmetry Breaking, Physica A263 (1999) 528-539.

[6] D. Ruelle, Gaps and New Ideas in our Understanding of Nonequilibrium, Physica A263 (1999) 540-544.

[7] K. Ikeda, H. Daido, O. Akimoto, Optical Turbulence: chaotic behavior of transmitted light from a ring cavity, Phys. Rev. Lett. 45 (1980) 709-712.

[8] V.V. Zverev, B.Y. Rubinstein, Chaotic oscillations and noise transformations in a simple dissipative system with delayed feedback, J. Statist. Phys. 63 (1991) 221-239.

[9] V.V. Zverev, The Fractal Structure of Invariant Distributions for Dissipative Random Mappings, Applied Nonlinear Dynamics (Izvestija Vuzov, Prikl. Nelin. Dinam. - Saratov, Russia) 3 (1995) 62-72.

[10] V.V. Zverev, On the conditions for the existence of fractal domain integrals, Theoretical and Mathematical Physics 107 (1996) 419-426.

[11] Surendra Singh, G.S. Agarwal, Chaos in coherent two-photon processes in a ring cavity, Optics Communications 47 (1983) 73-76.

[12] V.V. Zverev, On the origination of the chaotic attractor in nuclear spin motion in ferromagnet, Applied Nonlinear Dynamics (Izvestija Vuzov, Prikl. Nelin. Dinam. - Saratov, Russia) 1 (1993) $72-82$.

[13] V.V. Zverev, Modeling of chaos and noise conversion in magnetic systems by means of maps with nonlinear rotations, in Non-Linear Electromagnetic Systems, eds. V. Kose, J. Sievert, Studies in Applied Electromagnetics and Mechanics, 13 (IOS Press, 1998), pp.139-142.

[14] V.L. Safonov, V.V. Zverev, Dynamical chaos of collective oscillations in magnets, Physics of the Solid State 36 (1994) 1058-1062.

[15] A.P. Kuznetsov, L.V. Turukina, E. Mosekilde, Dynamical Systems of Different Classes as Models of the Kicked Nonlinear Oscillator. Intern. J. of Bifurcation and Chaos 11 (2000) 1065-1077.

[16] A. Brissaud, U. Frisch, Solving linear stochastic differential equations. J. Math. Phys. 15 (1974) 524-534. 
[17] H. Haken, Advanced Synergetics. Instability, Hierarchies of Self-Organizing Systems and Devices, in Springer Series in Synergetics, 20, ed. H. Haken (Springer, 1983).

[18] V. V. Zverev, Chaos and transformations of stochastic processes in nonlinear systems with intensity dependent phase rotations, in Doors to the Future, Proc. Int. Conf. on Nonlinearity, Bifurcations and Chaos (Lodź, Poland, 1996) p. 260.

[19] F.W.J. Olver, Bessel Functions of Integer Orders, in Handbook of Mathematical Functions, eds. M. Abramowitz, I.A. Stegun (Dover, 1970).

[20] M. Gell-Mann, M.R. Goldberger, The formal theory of scattering, Phys. Rev. 91 (1953) 398-408.

[21] D.N. Zubarev, Non-equilibrium Statistical Thermodynamics (Nauka, 1971).

[22] V. V. Zverev, B.Y. Rubinstein, Multistability resulting from random rearrangements in a ring resonator with a nonlinear element, Optics and Spectroscopy 62 (1987) 519-522.

[23] M. Reed, B. Simon, Methods of Modern Mathematical Physics, V.1, Functional Analysis (Academic Press, 1975). 